\title{
Current Status of International Particle Physics Masterclasses
}

\author{
Uta Bilow ${ }^{a, *}$ and Kenneth Cecire ${ }^{a, b}$ \\ ${ }^{a}$ Institut für Kern- und Teilchenphysik, Technische Universität Dresden \\ Zellescher Weg 19, 01969 Dresden, Germany \\ E-mail: uta.bilowetu-dresden.de \\ ${ }^{b}$ Department of Physics, University of Notre Dame \\ 225 Nieuwland Science Hall, Notre Dame, IN 46556, USA \\ E-mail: kcecireand.edu
}

Until recently, the International Masterclasses (IMC) in Particle Physics have been based on hands-on analysis of data from the four big LHC experiments. During the last years there has been a spectacular broadening in the physics scope of particle physics masterclasses, now including measurements with Belle II data, with data from neutrino experiments and a newly proposed masterclass on searches for dark matter. In addition, a particle therapy masterclass has been developed, in order to highlight some of the benefits for society from the technology developed for particle physics research. In parallel to extending the physics scope, IPPOG is making efforts to extend the geographical reach of masterclasses, and it is encouraging to see new institutes and countries joining each year. The IMC program is the flagship activity of IPPOG, the International Particle Physics Outreach Group.

40th International Conference on High Energy Physics - ICHEP2020

July 28-August 6, 2020

Prague, Czech Republic (virtual meeting) 


\section{Introduction}

International Masterclasses in particle physics have grown since inception to reach approximately 60 countries with daylong masterclasses held for over 10,000 high school students and, often, their teachers, at over 220 research facilities around the world. Such a masterclass is a way to make these students into "particle physicists for a day." A few to several hundred students at a university or laboratory interact with physicists in masterclasses which share key elements:

- Introductory presentation on the Standard Model and current research

- Facilities or laboratory tour

- Analysis of authentic experimental high-energy physics data

- Discussion of results

- Scheduled videoconference with other masterclass institutions analyzing data from the same experiment, moderated by physicists at CERN, Fermilab, KEK, GSI, or TRIUMF.

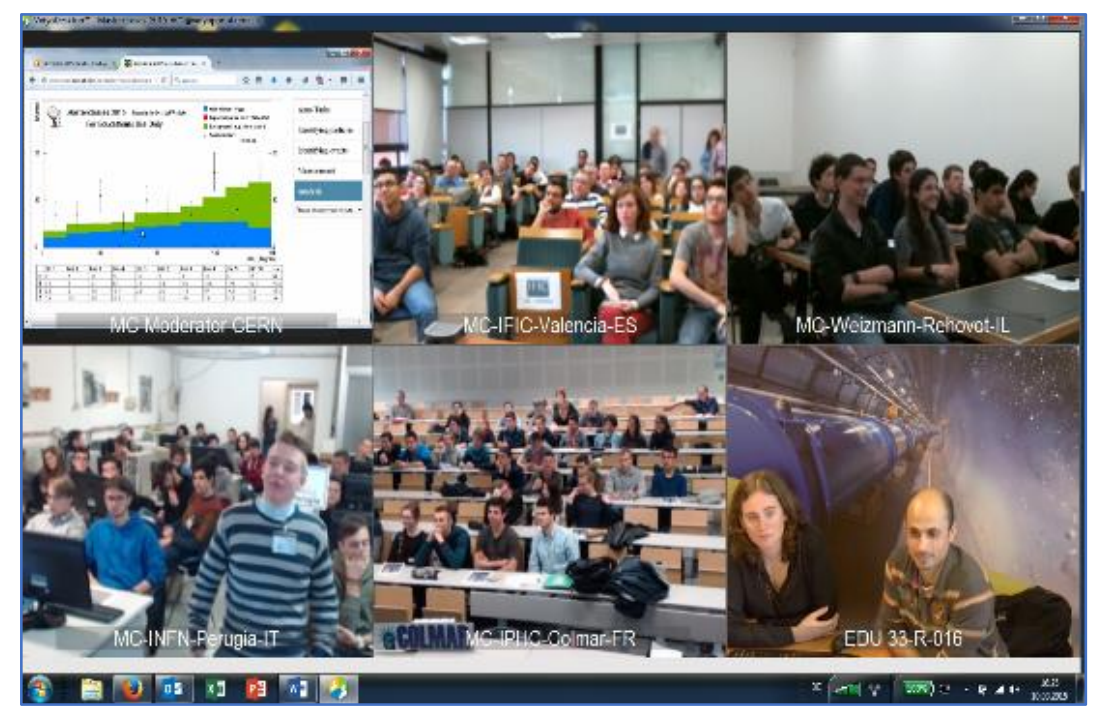

Figure 1: Masterclass videoconference for ATLAS W-path measurement.

\section{Measurements}

The heart of a particle physics masterclass - and what makes it a masterclass - is an authentic measurement from experimental data by students tutored by particle physicists. These data come from a few specific high-energy experiments.

Masterclass measurements share common elements. First, students examine data from event displays to make visual determinations based on the events and extract further data from them. Students work in pairs on some fixed set of 50-100 events. These teams of two can divide the work and check each other to maintain quality of interpretation. The results from the teams are combined to create plots or other representations from which the whole masterclass can draw conclusions. These are combined and compared in the videoconferences. [1]

\subsection{ATLAS Masterclass measurements}

There are two distinct measurements offered by International Masterclasses for ATLAS. 


\subsubsection{ATLAS I: Z-path}

In the ATLAS Z-path masterclass, students use the Java-based HYPATIA event display program, based on the ATLANTIS event viewer used in the ATLAS experiment. Pairs of students search through sets of 50 ATLAS events for decays which result in dilepton, diphoton, or fourlepton signatures. Students select the appropriate tracks for analysis and inclusion in mass plots. HYPATIA calculates the invariant masses and stores them in a text file; students then upload these to an online tool, OPloT, which creates the mass plots for examination and discussion.

The results in OPloT are organized into multiple available mass plots. Mainly, the diplepton events are organized around resonances for $Z^{0}, \mathrm{~J} / \Psi$, and $\mathrm{Y}$ particles, with the data pre-selected to emphasize the $Z^{0}$ boson. In addition, Monte Carlo data is added in to give suggestions of $Z$ ' and graviton signals in order that students might get a glimpse of the discovery process. Diphoton and four-lepton plots are also prominent but show no real peaks. Students doing these analyses learn not only about the ATLAS experiment but also concepts of statistics, modeling, and signal significance. [2]

\subsubsection{ATLAS II: W-path}

In the ATLAS W-path measurement, students use another variant of ATLANTIS to analyze leptonic W boson decays as well as WW pairs. Similar to the ATLAS Z-path, students work in pairs with sets of 50 events.

In their examination of $\mathrm{W}$ events, students ascertain the charge of the visible lepton to determine whether the parent particle was a $\mathrm{W}^{+}$or a $\mathrm{W}^{-}$. This data is combined in the masterclass to find a $\mathrm{W}^{+}$to $\mathrm{W}^{-}$ratio, which gives insight into the structure of the colliding protons from which the parent particles were formed.

Students examine WW events to find the azimuthal opening angle between visible leptons. Plotting this angle in a histogram provides insight into the Higgs discovery process at CERN. [3]

\subsection{CMS WZH measurement}

The main contribution from the Compact Muon Solenoid (CMS) experiment in the LHC is the CMS WZH measurement. All of the elements supporting this measurement are online. Pairs of students examine sets of 100 events in iSpy-webgl, a JavaScript program that runs in the browser of an Internet-connected computer. Students examine single lepton (plus missing $\mathrm{E}_{\mathrm{T}}$ ), dilepton, and four-lepton events. They determine $\mathrm{W}^{+} \mathrm{vs}$. $\mathrm{W}^{-}$events, dilepton invariant masses, and four-lepton invariant masses in iSpy-webgl. They transfer these results to an online analysis tool, the CMS Instrument for Masterclass Analysis (CIMA). In CIMA, students can find the $\mathrm{W}^{+}$to $\mathrm{W}^{-}$ and electron-to-muon ratios from combined results; CIMA also constructs combined dilepton mass histograms from which students can find resonances for $\mathrm{J} / \Psi, \mathrm{Y}$, and $\mathrm{Z}^{0}$ decays and fourlepton histograms which reveal $\mathrm{Z} \gamma^{*}, \mathrm{ZZ}$, and possible Higgs events. [4]

\subsection{LHCb Masterclass measurement}

In the LHCb masterclass, students search for decays of the $\mathrm{D}^{0}$ meson to kaons plus pions in the event display. Students can then create a mass plot to find the $\mathrm{D}^{0}$ invariant mass. In the next stage, have students measure displaced vertices of the $\mathrm{D}^{0}$ decays plus background, convert these to decay times, and then plot in a histogram to measure the lifetime from the resulting exponential decay curve. [5]

\subsection{ALICE Masterclass measurements}

There are two current ALICE masterclass measurements and another under development. 


\subsubsection{ALICE I: Looking for Strange Particles measurement}

The ALICE experiment offers two masterclass measurements. The most often used is called Looking for Strange Particles. Students examine event displays to find V0 particles-neutral strange particles such as strange kaons, lambdas, and anti-lambdas that are not directly observed but decay promptly into pairs of charged particles. Students can also find evidence of a negative $\mathrm{Xi}$ particle that decays into a pion and a lambda. From these decays, students can make plots to calculate the masses of the V0 particles; however, the number of events available of visual analysis is kept small. After working from the event display, students can use the software to create more detailed histograms from many more events, making appropriate subtractions of background and fitting the plots to polynomical functions. [6]

\subsubsection{ALICE II: Nuclear Modification Factor measurement}

The ALICE nuclear modification factor measurement is specifically directed to highlight collisions in the LHC heavy ion program. The nuclear modification factor, $\mathrm{R}_{\mathrm{AA}}$, describes the ratio of yield from $\mathrm{Pb}-\mathrm{Pb}$ collisions to the yield from an equivalent number of single proton-proton collisions. If $\mathrm{R}_{\mathrm{AA}}=1$, particle productioon in $\mathrm{Pb}-\mathrm{Pb}$ and proton-proton collisions can be said to be the same. A deviation from unity can be thought of as a measure of the effect of the quarkgluon plasma created in the $\mathrm{Pb}-\mathrm{Pb}$ collision on the particle yield. Students first examine data through a visual event display and establish $\mathrm{R}_{\mathrm{AA}}$ by counting tracks. There are a lot of tracks in a $\mathrm{Pb}-\mathrm{Pb}$ collision, so students then switch to a ROOT-based large scale analysis of $\mathrm{R}_{\mathrm{AA}}$ as a function of transverse momentum. Students use concepts of particle momentum and collision centrality and discover jet suppression by the quark-gluon plasma in the results of their analysis. [7]

\subsubsection{ALICE Masterclass update}

There are two important areas of progress for ALICE masterclasses. First, ALICE is in the process of adding a third measurement for the study of $\mathrm{J} / \Psi$ decays to electron-positron pairs in the ALICE time projection chamber (TPC). Students identify electron tracks by their energy loss, make a particle identification histogram, define their particle identification selections, make inverse mass histograms, and extract the $\mathrm{J} / \Psi$ yield. Students learn to use concepts of particle decay, invariant mass, and combinatorial backgroud to get their results.

In additon, while ALICE masterclass measurements have historically required ROOT, there is now a standalone application for all three ALICE measurements. It is easy to install and runs in Windows, Linux, and a virtual box. [8]

\subsection{MINERvA neutrino measurement}

One of the notable new additions to International Masterclasses is from the MINERvA neutrino detector at Fermilab. This was one of the first two non-LHC measurements to be adopted by International Masterclasses. In this measurement, muon neutrinos interact with a carbon target. Students visually search for and study interactions in which a proton and a muon are produced from a neutrino interacting with the carbon nucleus. In summing the momenta of the protons and muons over many events, they can infer the initial momentum distribution of the neutrinos, discover Fermi motion, and use the uncertainty principle to infer the size of the carbon nucleus as a test of two models of the interaction, one in which the neutrino interacts with free neutrons and the other interacting with the neutrons in a Fermi gas. [9]. 


\subsubsection{Further neutrino masterclass development}

In addition to the MINERvA measurement, QuarkNet staff in the U.S. and physicists are working on development of the following measurements: MicroBooNE, NoVA, and a DUNE simulation of a supernova signal. Ultimately, a major goal is a DUNE masterclass when the experiment is running and taking data.

\subsection{Belle II measurement}

The Belle II masterclass shows students how to code B-physics analysis. Students describe decays, make simple cuts, and "discover" particles using the visual code editor Blockly. This can be run from the web or from a downloaded virtual machine. There are some six million clean reconstructed events for students to analyze, including fitting peaks and determining widths. [10]

\subsection{Particle Therapy Masterclass}

In the Particle Therapy masterclass, students use a downloaded program called matRad to examine a simulated liver condition and plan treatment. Students write and then discuss treatment plans. The Particle Therapy Masterclass highlights some of the benefits for society of technology developed for particle physics research. It is particularly in demand in Latin America, which helps to extend International Masterclasses to new groups of students. [11]

\subsection{DarkSide Masterclass}

Members of the DarkSide dark matter search experiment at Gran Sasso are developing a masterclass for testing in International Masterclasses 2021. DarkSide searches for WIMPs in a dual phase argon time projection chamber. Students are given data in spreadsheet format. They run an anlaysis of some 20,000 events, make cuts, and look for rare WIMP candidate signals. [12]

\section{Conclusion}

The current pandemic has had a significant effect on International Masterclasses. It is to be expected that IMC 2021 will be affected as well but less so. There will be ample opportunities for physicists to be of help in reaching high school students with particle physics data analysis.

\subsection{Impact of the COVID-19 pandemic on International Masterclasses}

International Masterclasses 2020 were strongly affected by the onset and spread of the COVID-19 pandemic. By March 18, all IMC videoconferences were suspended. Only 25\% of masterclasses were completed.

The response was threefold:

- Staff created a simplified CMS analysis using only muon events (all else to be considered background) and with increased online support. This effort, the Big Analysis of Muons in CMS (BAMC) was conducted in April and May 2020 with significant teacher support for students and large webinars for lectures and masterclass videoconferences. [13]

- A number of groups did Masterclass@home, which was simply masterclasses delivered online to groups of individual students. Lectures and analysis were spread over two afternoons. 
- To give groups that had done a masterclass either online or in-person but had not had a chance for a videoconference, IMC coordination held some "make-up" videoconferences in June, with low turnout.

\subsection{Becoming involved}

There are a number of ways for physicists to become involved in International Masterclasses and become a greater part of particle physics education and outreach.

If one's institution participates in International Masterclasses, help is always welcome with organization, giving presentations, tutoring students as they perform data analysis, and leading discussion of results. If there is no masterclass at that institution, the physicist can start one. The zeroth step would be to contact the authors of this paper. [14] [15]

If the physicist works at an institution where IMC videoconferences are moderated-CERN, Fermilab, KEK, GSI, or TRIUMF - then becoming a masterclass videoconference moderator is a great opportunity to impact students. Training is provided and moderators work in pairs so that a new moderator usually has a "veteran" partner. Generally, a moderator takes on 2-3 "shifts."

\section{References}

[1] International Masterclasses website, https://www.physicsmasterclasses.org.

[2] ATLAS Z-path masterclass website, https://atlas.physicsmasterclasses.org/en/zpath.htm.

[3] ATLAS W-path masterclass website, https://atlas.physicsmasterclasses.org/en/wpath.htm.

[4] CMS masterclass website, https://cms.physicsmasterclasses.org/cms.html.

[5] LHCb masterclass website, https://hcb-public.web.cern.ch/en/LHCb-outreach/masterclasses/en/.

[6] ALICE Looking for Strange Particles masterclass website, https://alice-masterclass.web.cern.ch/.

[7] ALICE Nuclear Suppression Factor masterclass website, http://www-alice.gsi.de/masterclass/.

[8] ALICE GitHub page, https://github.com/zwound40/JpsiMasterclass.

[9] MINERvA neutrino masterclass website, https://indico.fnal.gov/event/22340/.

[10] Belle II masterclass website, https://belle2.ijs.si/public/.

[11] Particle Therapy masterclass website, https://indico.cern.ch/event/840212/.

[12] DarkSide website, https://indico.cern.ch/event/840212/.

[13] BAMC teacher launch page, https://quarknet.org/content/bamc-teacher-launch-page.

[14] International Masterclasses particle physics institutes page, http://www.physicsmasterclasses.org/index.php?cat=country.

[15] International Masterclasses organisation-introduction page, http://www.physicsmasterclasses.org/index.php?cat=local_organisation\&page=orga_intro. 